\title{
Dissecting Ubiquitin Signaling with Linkage-Defined and Protease Resistant Ubiquitin Chains**
}

\author{
Tatjana Schneider, Daniel Schneider, Daniel Rösner, Saurav Malhotra, Franziska Mortensen, \\ Thomas U. Mayer,* Martin Scheffner,* and Andreas Marx*
}

\begin{abstract}
Ubiquitylation is a complex posttranslational pro tein modification and deregulation of this pathway has been associated with different human disorders. Ubiquitylation comes in different flavors: Besides mono ubiquitylation, ubiquitin chains of various topologies are formed on substrate proteins. The fate of ubiquitylated proteins is determined by the linkage type of the attached ubiquitin chains, however, the underlying mechanism is poorly characterized. Herein, we describe a new method based on codon expansion and click chemistry based polymerization to generate linkage defined ubiquitin chains that are resistant to ubiquitin specific pro teases and adopt native like functions. The potential of these artificial chains for analyzing ubiquitin signaling is demon strated by linkage specific effects on cell cycle progression.
\end{abstract}

$M$ any, if not all, eukaryotic proteins are subjected to covalent modification by ubiquitin (ubiquitylation).$^{[1]}$ Indeed, numerous fundamental cellular processes are regulated by ubiquitylation and, accordingly, dysfunctional ubiquitylation has been associated with various human disorders, including cancer and neurodegenerative diseases. ${ }^{[2]}$ Ubiquitylation is mediated by the concerted action of at least three classes of enzymes: E1 ubiquitin activating enzymes, E2 ubiquitin conjugating enzyme, and E3 ubiquitin ligases. ${ }^{[3]}$ Mono ubiq uitylation refers to the covalent attachment of a single ubiquitin moiety to a substrate protein through an isopeptide bond between the carboxyl group of the $\mathrm{C}$ terminal glycine of ubiquitin and an $\varepsilon$ amino group of a lysine residue of the substrate. In many cases, the added ubiquitin moiety can then serve as a ubiquitylation substrate, thereby resulting in the formation of ubiquitin chains on substrate proteins (poly

$\left[{ }^{[*}\right]$ T. Schneider, ${ }^{[+]}$Dr. D. Schneider, ${ }^{[+]}$D. Rösner, ${ }^{[+]}$S. Malhotra, F. Mortensen, Prof. Dr. T. U. Mayer, Prof. Dr. M. Scheffner, Prof. Dr. A. Marx

Departments of Chemistry and Biology, Konstanz Research School Chemical Biology, University of Konstanz

Universitätsstrasse 10, 78457 Konstanz (Germany)

E mail: thomas.u.mayer@uni konstanz.de martin.scheffner@uni konstanz.de andreas.marx@uni konstanz.de

$\left.{ }^{+}\right]$These authors contributed equally to this work.

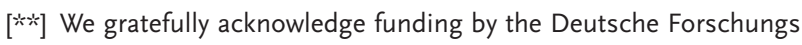
gemeinschaft within the SFB 969 "Chemical and Biological Principles of Cellular Proteostasis" and the Carl Zeiss Stiftung (stipend to T.S.). We also acknowledge the Konstanz Research School Chemical Biology for financial support. We thank Dr. M. Rubini for discussion and critical reviewing of the manuscript. ubiquitylation). Since ubiquitin has seven lysine residues (K6, $\mathrm{K} 11, \mathrm{~K} 27, \mathrm{~K} 29, \mathrm{~K} 33, \mathrm{~K} 48$, and K63) and each of these, as well as the $\alpha$ amino group of the $\mathrm{N}$ terminal methionine, can be used for chain formation, ${ }^{[4]}$ poly ubiquitylation engenders a great diversity of possible signals. Typically, the lysine residues, as well as the $\mathrm{N}$ terminal methionine, are used in a site specific manner by the conjugation machinery, thereby resulting in the formation of homotypic ubiquitin chains. The actual linkage type within such chains determines the fate of poly ubiquitylated proteins. The best characterized function, targeting proteins to the $26 \mathrm{~S}$ proteasome for degradation, is achieved by modifying the substrate with a ubiquitin chain of at least four ubiquitin moieties linked through K48. ${ }^{[5]}$ Furthermore, K11 and possibly K29 linked ubiquitin chains also serve as signals for the proteasomal pathway. ${ }^{[6]}$ In contrast, K63 linked ubiquitin chains play non proteasomal roles; for example, in endocytosis, DNA repair, and signal transduction; ${ }^{[7]}$ and linear ( $\mathrm{N}$ terminal linked) ubiquitin chains are involved in NF $\kappa \mathrm{B}$ activation. ${ }^{[8]}$ The existence of K6, K27, and K33 linked poly ubiquitylated proteins has been reported, but their function(s) remains elusive. ${ }^{[4 b]}$

A major obstacle in understanding how the linkage impacts the fate of modified proteins is the difficulty in generating linkage specific chains in quantities sufficient for subsequent investigations. Since they are not accessible by molecular cloning, ubiquitin chains have been synthesized enzymatically, which is so far only possible for K11, K48, and K63 linked chains, as well as linear chains. ${ }^{[4 a, 9]}$ Further more, the applicability of these ubiquitin chains, for example, in cell extracts, is significantly limited by the fact that these chains are rapidly dismantled by de ubiquitylating enzymes (DUBs). ${ }^{[10]}$ A potential solution to the latter problem was recently provided by using a genetically encoded ubiquitin mutant UbL73P to enzymatically assemble ubiquitin chains that are resistant to DUBs. ${ }^{[1]}$ To address the challenge of synthesizing ubiquitin chains of any linkage type, chemical approaches including thiol ene coupling, ${ }^{[12]}$ silver mediated chemical condensation, ${ }^{[13]}$ and chemical synthesis combined with isopeptide chemical ligation ${ }^{[14]}$ have been described (reviewed in [15]). Furthermore, the groups of Brik and Strieter have synthesized bi functional ubiquitin variants to produce chains through polymerization. ${ }^{[12,14 \mathrm{a}]}$ However, the linkages between the individual ubiquitin moieties are cleavable by DUBs. Thus, to shed light on the function of individual ubiquitin chains, additional approaches that com bine DUB resistance and easy access to any linkage type desired are much needed. Recently, we and others established a means to generate mono ubiquitylated substrates and ubiquitin dimers linked through a triazole by combining the 
incorporation of unnatural amino acids into proteins and subsequent bond formation through bioorthogonal chemis try. ${ }^{[16]}$ To this end, the azide functionalized methionine analogue azidohomoalanine (Aha, Figure 1a) was incorpo rated at the $\mathrm{C}$ terminus of ubiquitin and a distinct lysine residue of the substrate was replaced by an alkyne function alized lysine analogue (Plk, Figure 1a), thereby enabling lysine residues $11,27,29$, and 48 were chosen as conjugation sites, since K11 and K48 linked ubiquitin chains have well characterized features, whereas little is known about the linkage types K27 and K29 (Figure 1 b). All four variants were expressed in methionine auxothrophic E. coli B834 (DE3) cells in minimal medium containing Aha to perform SPI. To allow ACS, we used a helper plasmid based on the pEVOL

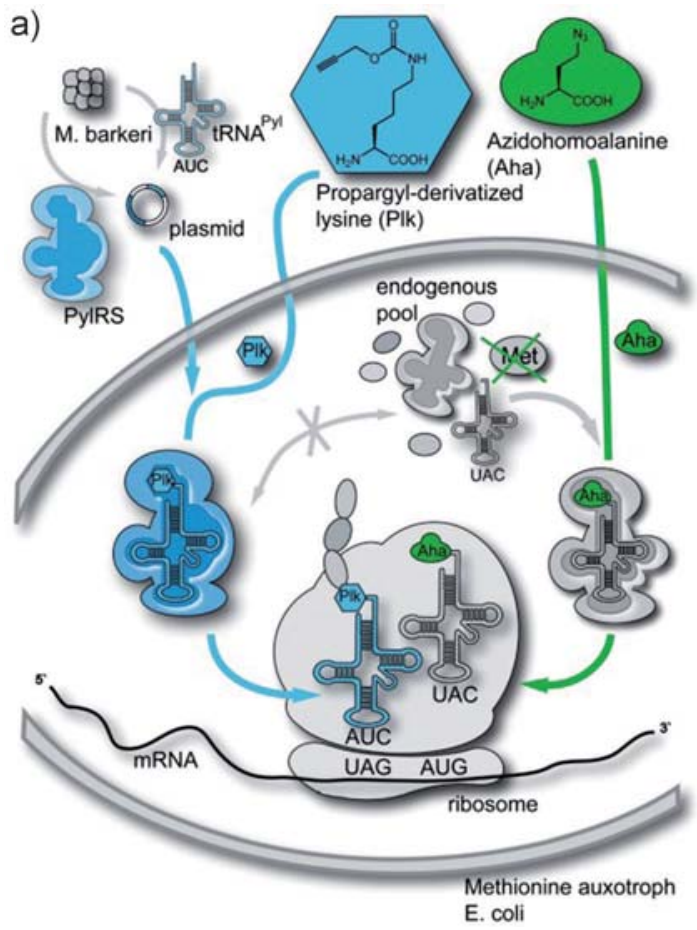

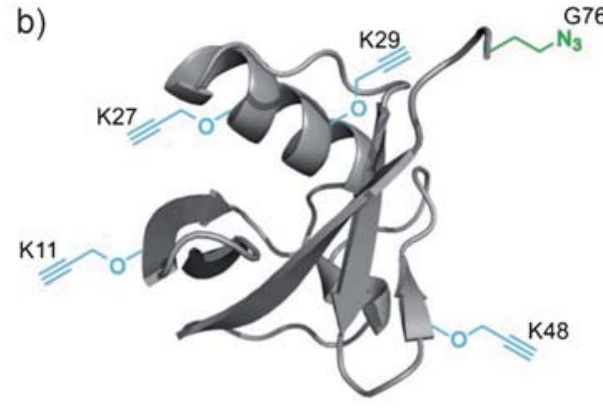

c)

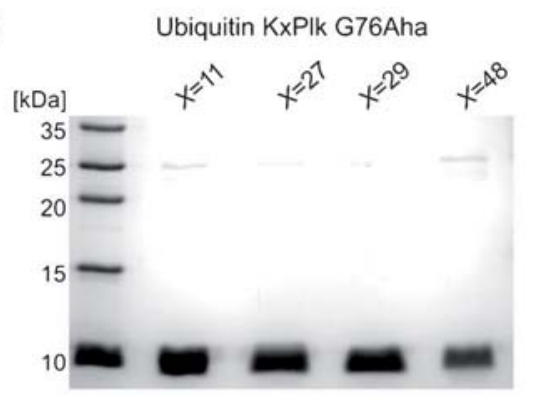

Figure 1. The two unnatural amino acids necessary for click reaction can be incorporated simultaneously and site specifically into ubiquitin. a) Concept of the strategy used. The gene of interest is expressed in methionine auxtrophic E. coli B834 (DE3) cells. The minimal medium used contains no methionine but rather azidohomo alanine (Aha; green) and a propargyl derivatized lysine (Plk; blue). Aha is utilized by the endogenous MetRS and tRNA ${ }^{\text {Met }}$ and thus incorporated instead of methionine into the proteome through SPI. The PyIRS and tRNA ${ }^{\text {Pyl }}$ (blue) from Methanosarcina barkeri are expressed on an accessory plasmid and accept Plk as substrate, thus enabling the incorporation of an alkyne opposite an amber stop codon (ACS). b) A schematic structure of the four ubiquitin KxPlk G76Aha variants generated. The four alkyne moieties, incorporated separately in each variant, are shown in blue and are labeled according to the lysine residue replaced. The $C$ terminal azide group present in each variant is shown in green. c) Analysis of the purified ubiquitin variants by SDS PAGE and Coomassie blue staining.

ubiquitylation by $\mathrm{Cu}^{\mathrm{I}}$ catalyzed azide alkyne cycloaddition ("click chemistry"). We now present a method to generate DUB resistant ubiquitin chains of any defined linkage type by using a bifunctional ubiquitin. Our approach is generally applicable without requiring sophisticated equipment for chemical synthesis. Conceptually, the two unnatural amino acids necessary for the click reaction, Aha and Plk, are incorporated within a single ubiquitin moiety through the simultaneous application of amber codon suppression (ACS) and selective pressure incorporation (SPI; Figure 1a). Sub sequent click chemistry based polymerization enables effi cient formation of homotypic ubiquitin polymers.

To match the native linkage sites, Aha was incorporated instead of the $\mathrm{C}$ terminal glycine of Ubiquitin and a single lysine was replaced site specifically by Plk. As case studies, construct and added Plk to the medium ${ }^{[17]}$ After purification via the GST tag and subsequent thrombin cleavage, ${ }^{[16 b]}$ full length protein con taining both unnatural amino acids was obtained for all four constructs in yields between $0.5 \mathrm{mg}$ and $2.0 \mathrm{mg}$ per liter expres sion culture (Figure 1c). With these bifunctional ized proteins in hand, we initially investigated the polymerization capacity of the ubiquitin variants K11Plk G76 Aha, K27Plk G76Aha, and K29Plk G76Aha. In all cases, efficient for mation of oligomers/ polymers was detected by SDS PAGE (Fig ure $2 \mathrm{a})$. For ubiquitin K27Plk G76Aha and ubiquitin K29Plk G76 Aha, large polymers up to 30 mers were observed, thus indicat ing a highly efficient reaction. For the ubiqui tin variants with Plk at positions $\mathrm{K} 11$ and $\mathrm{K} 48$ (Figure 2a, Figure S2 in the Supporting Informa tion), slightly lower efficiencies with respect to longer polymers were found.

Next, in line with recent work by Brik and co workers, ${ }^{[18]}$ the potential to assemble ubiquitin chains site specifically on target proteins in a one pot reaction was investigated (Fig ure $2 \mathrm{~b}, \mathrm{c}$ ). As proof of principle, $\mathrm{His}_{6}$ tagged human DNA polymerase beta $(\mathrm{Pol} \beta) \mathrm{K} 61 \mathrm{Plk},{ }^{[16 \mathrm{~b}]}$ a protein exclusively functionalized with an alkyne group and thus a substrate for poly ubiquitylation was mixed with the ubiquitin variant K11Plk G76Aha at different concentrations of ubiquitin and click reaction was performed. As expected, in the absence of Pol $\beta$ K61Plk, efficient formation of free ubiquitin chains was observed, whereas Pol $\beta$ K61Plk alone resulted in a single band. When both proteins were mixed, multiple bands at higher molecular weights were detected that increased in 
a)

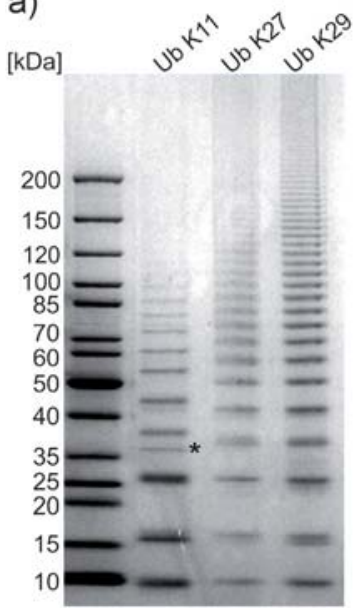

b)

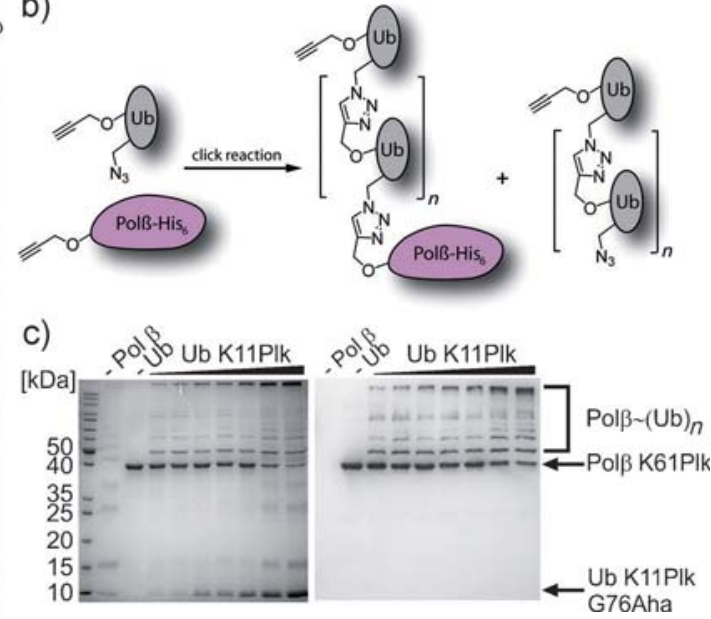

Figure 2. Ubiquitin variants can form long polymers, both free in solution and on substrate proteins upon click reaction. a) Analysis of polymer formation for three ubiquitin KxPIk G76Aha variants after click reaction by SDS PAGE and Coomassie blue staining. The asterisk indicates impurity present in the ubiquitin (Ub) K11Plk G76Aha preparation. b) Schematic depiction of the one pot reaction between bifunctional ubiquitin and alkyne functionalized Pol $\beta$. Upon click reaction, both free ubiquitin chains and ubiquitylated Pol $\beta$ are formed. c) The products of the click reaction when using different amounts of ubiquitin K11Plk G76Aha and Pol $\beta$ K61Plk were analyzed either by SDS PAGE and Coomassie blue staining (left panel) or by Western Blot (right panel) with a His ${ }_{6}$ tag specific antibody.

intensity with the amount of ubiquitin added. To investigate which bands corresponded to poly ubiquitylated Pol $\beta$, West ern blot analysis with an antibody against the His ${ }_{6}$ tag was performed. Highly poly ubiquitylated Pol $\beta$ species, along with mono and di ubiquitylated species, were detected even at low ubiquitin concentrations, thus demonstrating that poly ubiquitin chains can easily be attached to target proteins in a one pot reaction.

We also performed a similar reaction with exclusively alkyne functionalized ubiquitin (K11Plk G76) as the substrate for poly ubiquitylation (Figure S1a). As expected, with increasing amounts of K11Plk G76, the length of the ubiquitin chains decreased, thus indicating ubiquitylation with ubiquitin K11Plk G76, which serves as a terminator for polymerization.

Since it was reported that naturally linked ubiquitin chains can be activated by E1 ${ }^{[13 a, 19]}$ we next investigated whether the artificial chains that bear a $\mathrm{C}$ terminal glycine at their proximal end are also activated by the E1 enzyme and are still substrates for the ubiquitylation machinery. To do so, an E6AP auto ubiquitylation assay was performed (Fig ure S1b,c). Indeed, we found that ubiquitin chains generated by click reaction can be activated and covalently attached to E6AP or other ubiquitin chains (resulting in the formation of longer oligomers).

We next investigated whether connecting the individual ubiquitin moieties with triazoles still results in chains with native like properties, as it has already been demonstrated for di ubiquitin by Mootz and co workers. ${ }^{[20]}$ For this purpose, an antibody that selectively binds K48 linked ubiquitin chains was employed (Figure S2). Indeed, K48 linked ubiquitin oligomers were detected while no signals could be observed for the other chain linkages.

To prove the potential of the artificially linked ubiquitin chains for investigating ubiq uitin signaling, we tested their effect on the activity of the $26 \mathrm{~S}$ proteasome in Xenopus laevis egg extracts, a model system for the functional analysis of cell cycle regulation by the ubiquitin proteasome system. We postu lated that if the artificial chains adopted the proper topology, they would be recognized by the $26 \mathrm{~S}$ proteasome or other factors required for proteasome mediated degradation but since the triazole linkage renders them refractory to the action of DUBs, they would stay bound to the proteasome, thereby competing with the degradation of natural substrates of the proteasome. To test this, the cell cycle dependent degradation of cyclin B was monitored. Since cyclin B is known to be targeted to the proteasome through K11 linked ubiquitylation, $\left.{ }^{[6]}\right]$ we used the artifi cially linked K11 chains. Furthermore, K27 linked and K29 linked chains were tested because it is currently unclear whether they are efficiently recognized by the proteasome and promote degradation of substrate pro teins. Cyclin B is efficiently degraded upon the addition of $\mathrm{Ca}^{2+}$, which leads to activation of the multisubunit E3 ligase Anaphase Promoting Complex/ Cyclosome through a signaling cascade that mimics fertiliza tion. ${ }^{[21]}$ Consistent with the fact that free monomeric ubiquitin is not recognized by the proteasome, the addition of mono meric ubiquitin K11Plk G76Aha did not influence the degradation of cyclin B (Figure $3 \mathrm{a}$ ). Remarkably, addition of the same amount (or even lower amounts; Figure S3) of K11 linked ubiquitin chains completely abolished the cal cium induced degradation of cyclin B. Furthermore, DNA morphology and spindle formation were assessed. After $\mathrm{Ca}^{2+}$ addition, buffer treated extracts displayed a typical interpha sic nuclear morphology (Figure 3c), thus confirming exit from meiosis upon cyclin B degradation. In contrast, extracts treated with K11 linked chains or the proteasome inhibitor MG262 displayed condensed chromatin and bipolar spindle structures (Figure $3 \mathrm{c}$ ), thus indicating perpetuation of the meiotic state owing to the inability to degrade cyclin B. These data demonstrate that the artificial ubiquitin polymers were physiologically functional in whole cell extracts. Notably, the addition of similar amounts of both K27 linked and K29 linked chains did not interfere with the degradation of cyclin B (Figure 3a,b). This result indicates that K27 linked and K29 linked chains are not bound or are only poorly bound by the proteasome, thus suggesting that they have cellular functions other than serving as a signal for protea somal degradation. Alternatively, in meiotic extracts, pro tein(s) may be missing that serve as shuttle factors to transport proteins modified by K27 linked or K29 linked ubiquitin chains to the proteasome.

In summary, we developed a straightforward and unique approach for the generation of ubiquitin containing two unnatural amino acids that render it suitable for bioorthog 
a)

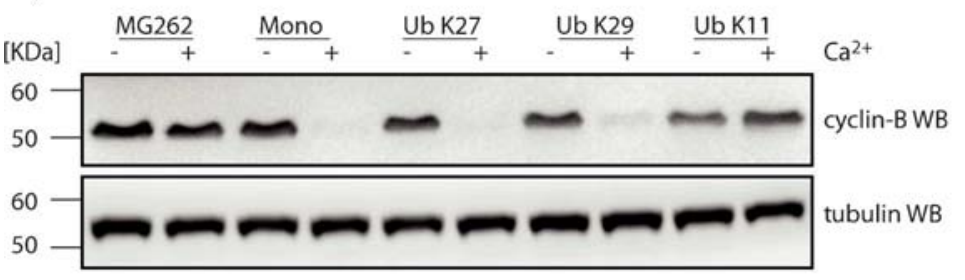

b)

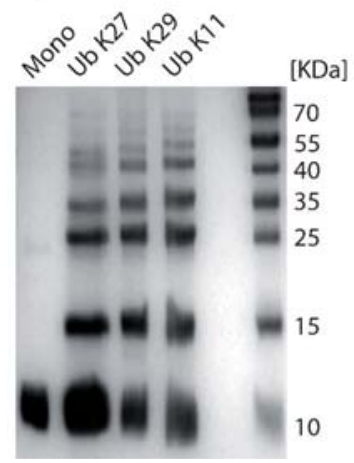

c)

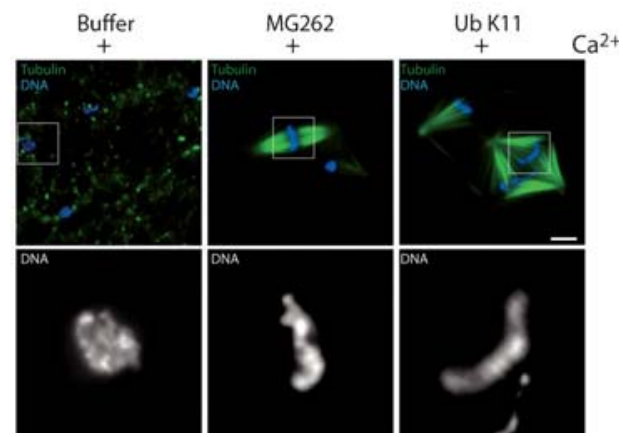

Figure 3. $\mathrm{K} 11$ linked chains prevent the degradation of cyclin B in Xenopus egg extracts. a) Upon calcium $\left(\mathrm{Ca}^{2+}\right)$ addition, cyclin $\mathrm{B}$ remains stable in extracts supple mented with $\mathrm{K} 11$ linked chains or MG262 but is efficiently degraded in extracts containing mono Ub K11Plk G76Aha, K27 linked chains, or K29 linked chains, as can be seen in the cyclin B Western Blot. Tubulin serves as a loading control. b) SDS PAGE of the ubiquitin preparations used for the Xenopus egg extract experiments followed by Coomassie blue staining. The amounts loaded in each lane correspond to the amounts used in (a). c) After $\mathrm{Ca}^{2+}$ addition, buffer treated extracts exit the meiotic state and display interphase chromatin (inset magnification shown in the lower panel) and no microtubule spindle structures, whereas extracts treated with MG262 or K11 linked chains show meiosis specific bipolar spindles (upper panels) and condensed chromatin (inset magnification, lower panels). Spindles and chromosomes were visualized by the addition of TRITC tubulin and DAPI, respectively.

onal polymerization. Glycine 76 was replaced by an azide, whereas an alkyne functionalized lysine derivative was incor porated site specifically in place of one of the seven natural lysine residues. For the four dual modified ubiquitin variants studied, yields in the milligram range were obtained and subsequent click chemistry based polymerization delivered linkage defined ubiquitin chains. Furthermore, we demon strate that our approach holds the potential to easily generate defined poly ubiquitylated target proteins in a one pot reaction. Importantly, the protease resistance of these chains enabled us to study linkage specific effects on meiotic progression in the complex biological system Xenopus laevis egg extract. Indeed, we discovered that unlike K11 linked chains, K27 and K29 linked chains do not serve as protea somal degradation signals. Furthermore, we provide evidence that ubiquitin chains can be activated and conjugated en bloc to substrates by the ubiquitylation machinery. Thus both features, namely a defined linkage type and resistance to de ubiquitylating enzymes, render these chains invaluable tools for dissecting the diversity of ubiquitin signaling in complex biological systems.
Keywords: click chemistry · codon expansion . posttranslational modification - ubiquitin chains . unnatural amino acids

[1] a) K. Haglund, I. Dikic, EMBO J. 2005, 24, 3353 3359; b) L. Spasser, A. Brik, Angew. Chem. Int. Ed. 2012, 51, 6840 6862; Angew. Chem. 2012, 124, 6946 6969; c) A. Hershko, A. Ciechanover, Annu. Rev. Biochem. 1998, 67, 425479.

[2] a) A. Ciechanover, P. Brundin, Neuron 2003, 40, 427 446; b) D. Hoeller, C. M. Hecker, I. Dikic, Nat. Rev. Cancer 2006, 6, 776788.

[3] a) M. H. Glickman, A. Ciechanover, Physiol. Rev. 2002, 82, 373 428; b) M. Scheffner, U. Nuber, J. M. Huibregtse, Nature 1995, 373, 8183.

[4] a) T. Kirisako, K. Kamei, S. Murata, M. Kato, H. Fukumoto, M. Kanie, S. Sano, F. Tokunaga, K. Tanaka, K. Iwai, EMBO J. 2006, 25, 4877 4887; b) J. Peng, D. Schwartz, J. E. Elias, C. C. Thoreen, D. Cheng, G. Marsischky, J. Roelofs, D. Finley, S. P. Gygi, Nat. Biotechnol. 2003, 21, 921926.

[5] J. S. Thrower, L. Hoffman, M. Rechsteiner, C. M. Pickart, EMBO J. 2000, 19, 94102.

[6] a) P. Chastagner, A. Israel, C. Brou, EMBO Rep. 2006, 7, 1147 1153; b) L. Jin, A. Williamson, S. Banerjee, I. Philipp, M. Rape, Cell 2008, 133, 653 665; c) M. Koegl, T. Hoppe, S. Schlenker, H. D. Ulrich, T. U. Mayer, S. Jentsch, Cell 1999, 96, 635 644.

[7] a) D. Komander, M. Rape, Annu. Rev. Biochem. 2012， 81， 203 229; b) R. L. Welchman, C. Gordon, R. J. Mayer, Nat. Rev. Mol. Cell Biol. 2005, 6, 599609.

[8] a) K. Iwai, Cell Cycle 2011, 10, 3095 3104; b) F. Ikeda, Y. L. Deribe, S. S. Skanland, B. Stieglitz, C. Grabbe, M. Franz Wachtel, S. J. van Wijk, P. Goswami, V. Nagy, J. Terzic, F. Tokunaga, A. Androulidaki, T. Nakagawa, M. Pasparakis, K. Iwai, J. P. Sundberg, L. Schaefer, K. Rittinger, B. Macek, I. Dikic, Nature 2011, 471, 637641.

[9] a) C. M. Pickart, S. Raasi, Methods Enzymol. 2005, 399, 21 36; b) M. Rape, Subcell. Biochem. 2010, 54, 107115 .

[10] F. E. Reyes Turcu, K. H. Ventii, K. D. Wilkinson, Annu. Rev. Biochem. 2009, 78, 363397.

[11] M. Békés, K. Okamoto, S. B. Crist, M. J. Jones, J. R. Chapman, B. B. Brasher, F. D. Melandri, B. M. Ueberheide, E. L. Denchi, T. T. Huang, Cell Rep. 2013, 5, 826838.

[12] V. H. Trang, E. M. Valkevich, S. Minami, Y. C. Chen, Y. Ge, E. R. Strieter, Angew. Chem. Int. Ed. 2012, 51, 13085 13088; Angew. Chem. 2012, 124, 1326213265.

[13] a) C. Castañeda, J. Liu, A. Chaturvedi, U. Nowicka, T. A. Cropp, D. Fushman, J. Am. Chem. Soc. 2011, 133, 17855 17868; b) E. K. Dixon, C. A. Castañeda, T. R. Kashyap, Y. Wang, D. Fushman, Bioorg. Med. Chem. 2013, 21, 3421 3429; c) S. Virdee, Y. Ye, D. P. Nguyen, D. Komander, J. W. Chin, Nat. Chem. Biol. 2010, 6, 750757.

[14] a) T. Moyal, S. N. Bavikar, S. V. Karthikeyan, H. P. Hemantha, A. Brik, J. Am. Chem. Soc. 2012, 134, 16085 16092; b) K. S. Kumar, S. N. Bavikar, L. Spasser, T. Moyal, S. Ohayon, A. Brik, Angew. Chem. Int. Ed. 2011, 50, 6137 6141; Angew. Chem. 2011, 123, 62616265 ; c) S. N. Bavikar, L. Spasser, M. Haj Yahya, S. V. Karthikeyan, T. Moyal, K. S. Ajish Kumar, A. Brik, Angew. Chem. Int. Ed. 2012, 51, 758 763; Angew. Chem. 2012, 124, 782 787.

[15] a) A. Brik, Bioorg. Med. Chem. 2013, 21, 3398 3399; b) H. P. Hemantha, A. Brik, Bioorg. Med. Chem. 2013, 21, 34113420. 
[16] a) S. Eger, M. Scheffner, A. Marx, M. Rubini, J. Am. Chem. Soc 2010, 132, 16337 16339; b) D. Schneider, T. Schneider, D. Rösner, M. Scheffner, A. Marx, Bioorg. Med. Chem. 2013, 21, 34303435 ; c) S. Sommer, N. D. Weikart, A. Brockmeyer, P. Janning, H. D. Mootz, Angew. Chem. Int. Ed. 2011, 50, 9888 9892; Angew. Chem. 2011, 123, 10062 10066; d) S. Eger, B. Castrec, U. Hübscher, M. Scheffner, M. Rubini, A. Marx, ChemBioChem 2011, 12, 28072812.

[17] T. S. Young, I. Ahmad, J. A. Yin, P. G. Schultz, J. Mol. Biol. 2010, 395,361374
[18] H. P. Hemantha, S. N. Bavikar, Y. Herman Bachinsky, N. Haj Yahya, S. Bondalapati, A. Ciechanover, A. Brik, J. Am. Chem. Soc. 2014, 136, 26652673

[19] a) O. N. Burchak, M. Jaquinod, C. Cottin, L. Mugherli, K. Iwai, F. Chatelain, M. Y. Balakirev, ChemBioChem 2006, 7, 1667 1669; b) S. van Nocker, R. D. Vierstra, J. Biol. Chem. 1993, 268, 2476624773.

[20] T. Dresselhaus, N. D. Weikart, H. D. Mootz, M. P. Waller, RSC Adv. 2013, 3, 1612216129.

[21] N. R. Rauh, A. Schmidt, J. Bormann, E. A. Nigg, T. U. Mayer, Nature 2005, 437, 10481052. 